\title{
Anti-Obesity Effects of Artemisia annua Extract in Zucker Fatty Rats and High-Fat Diet Sprague Dawley Rats through Upregulation of Uncoupling Protein 1
}

\author{
Eun-Yong Choi ${ }^{1}$, Chan Young Park ${ }^{1}$, Seong Hyun Ho², Su-Jin Park ${ }^{2}$, Donghyun Kim ${ }^{3,4}$, Byoungduck Han ${ }^{5, *}$, Seon-Hee Kim ${ }^{1, *}$ \\ 'Sungkyun Biotech Co., Ltd., Suwon; ${ }^{2}$ G\&P Bioscience Co. LTD., Goyang; Departments of ${ }^{3}$ Biomedical Sciences and ${ }^{4}$ Microbiology and Immunology, Seoul National \\ University College of Medicine, Seoul, ${ }^{5}$ Department of Family Medicine, Korea University Guro Hospital, Korea University College of Medicine, Seoul, Korea
}

Background: Obesity is a widespread disease and is caused mainly by excessive adipocyte differentiation and fat accumulation. Peroxisome proliferation-activated receptor $\gamma$ (PPAR $\gamma$ ) and CCAAT/enhancer-binding proteins (C/EBP) are major components for regulating adipocyte differentiation. Uncoupling protein 1 (UCP1) is a transmembrane protein that can convert white fat to brown adipose tissue. Artemisia annua L. has long been used in East Asia as an herbal drug for anti-oxidant, anti-bacterial, and anti-obesity purposes.

Methods: We investigated the effects of water extracts of $A$. annua (WEAA) in (3H10T1/2, a mesenchymal stem cell line, by measuring the level of intracellular fat accumulation and the expression of genes associated with adipocyte differentiation. We also evaluated anti-obesity effects ofWEAA in Zucker rats, a genetic model for the study of obesity, and in Sprague Dawley rats with high-fat diet (HFD)-induced obesity.

Results: In this study, WEAA reduced the expression levels of PPAR $\gamma$ and C/EBPa in C3H10T1/2 cells, as well as the expression of enzymes that regulate fatty acid metabolism. In the Zucker fatty rat model and the HFD-induced obesity rat model, WEAA significantly decreased adipogenic differentiation and white fat accumulation between the scapulae, in contrast to the brown fat that remained unchanged between the groups. A. annua suppressed the expression of the adipocyte differentiation-promoting genes, while increasing the expression of UCP1.

Conclusion: These results indicated that WEAA could reduce adipocyte differentiation and fat accumulation in in vitro and in vivo model systems, resulting in suppression of obesity and the occurrence of fatty liver due to a HFD.

Key words: Artemisia annua, Obesity, Adipogenesis, Uncoupling protein 1, Non-alcoholic fatty liver diseases

\author{
Received September 18, 2020 \\ Reviewed October 30, 2020 \\ Accepted December 2, 2020 \\ *Corresponding author \\ Seon-Hee Kim
}

(iD)

https://orcid.org/0000-0003-4079-2594

Sungkyun Biotech Co., Ltd.,

142-10 Saneop-ro 156beon-gil,

Gwonseon-gu, Suwon 16648, Korea

Tel: +82-31-299-4811

Fax: +82-31-739-5852

E-mail:shkim31@sungkyunbiotech.co.kr

${ }^{*}$ Co-Corresponding author

Byoungduck Han

https://orcid.org/0000-0003-2830-1174

Department of Family Medicine, Korea University Guro Hospital, Korea University College of Medicine, 148 Gurodong-ro,

Guro-gu, Seoul 08308, Korea

Tel: +82-2-2626-1547

Fax: +82-2-837-3966

E-mail: arybury@korea.ac.kr

\section{INTRODUCTION}

Obesity, a medical condition that involves accumulation of excessive body fat, is a widespread disease all over the world and remains one of the largest issues in health care. ${ }^{1}$ Diverse medical dis- eases are associated with obesity, such as hyperlipidemia, type 2 diabetes, atherosclerosis, hypertension, and cardiovascular disease. ${ }^{2-5}$ Obesity is caused by an increase in adipocyte size (hypertrophy) and production of new adipocytes (hyperplasia). ${ }^{6}$ Understanding the regulation of adipocytes can provide strategies for treating obe-

Copyright (C) 2021 Korean Society for the Study of Obesity

(a) This is an Open Access article distributed under the terms of the Creative Commons Attribution Non-Commercial License (https://creativecommons.org/licenses/by-nc/4.o/) which permits unrestricted non-commercial use, distribution, and reproduction in any medium, provided the original work is properly cited. 
sity and obesity-related diseases.

Adipocyte differentiation is a process of cell differentiation in which preadipocytes become adipocytes. ${ }^{7}$ Differentiation is regulated by diverse intracellular proteins, including adipogenic and lipogenic-related proteins and other metabolic signal transduction proteins. ${ }^{8,9}$ Peroxisome proliferation-activated receptor $\gamma(\operatorname{PPAR} \gamma)$ and CCAAT/enhancer-binding proteins $(\mathrm{C} / \mathrm{EBP} \alpha,-\beta$, and $\delta)$ are major components that work cooperatively to control adipocyte differentiation. ${ }^{10,11}$ Uncoupling protein 1 (UCP1), also called thermogenin, is a transmembrane protein found in the mitochondria of brown adipose tissue and is associated with heat generation by non-shivering thermogenesis. ${ }^{12,13}$ Increased UCP1 expression is closely related to anti-obesity properties in mice ${ }^{14,15}$ and can be a useful defense mechanism against obesity by stimulating intracellular energy consumption and regulating adipocyte differentiation.

Artemisia annua, also known as annual mugwort, sweet wormwood, or sweet annie, is an annual herb belonging to the family Asteraceae and is commonly found in parts of Asia, including China and Korea. Traditionally, A. annua has been used to treat heat and chills in China. ${ }^{16}$ Artemisinin and its derivatives, which are components of $A$. annua, have been widely used as anti-malarial drugs and are effective anti-cancer and anti-viral agents. ${ }^{17,18}$ Artemisinic acid, isolated from $A$. апnua, inhibits adipocyte differentiation in human adipose tissue-derived mesenchymal stem cells ${ }^{19}$ while essential oil from $A$. annua has anti-adipocyte differentiation effects in 3T3-L1 cells. $^{20}$

However, the anti-obesity effects of $A$. annua are unknown in C3H10T1/2 cells and transgenic animal models. In this study, we investigated the effects of water extracts of A. annua (WEAA) in C3H10T1/2 cells by measuring the level of intracellular fat accumulation and the expression of genes associated with adipocyte differentiation. We also evaluated anti-obesity effects in Zucker rats, a genetic model for the study of obesity, and in Sprague Dawley (SD) rats with high-fat diet (HFD)-induced obesity.

\section{METHODS}

\section{Preparation of $\boldsymbol{A}$. annua extracts and Garcinia cambogia extracts}

A. апnиa was purchased from the Korean commercial market.
Chopped A. annua was extracted with water for 4 hours at $85^{\circ} \mathrm{C}$ and filtered through filter paper (Whatman 3; GE Healthcare, Chicago, IL, USA). The filtrate was concentrated to 50 brix at a temperature below $60^{\circ} \mathrm{C}$, and this concentrate was used for the in vitro assay. For the in vivo experiment, the concentrate was mixed with $30 \%$ dextrin and then powdered with a spray dryer. The $A$. annua content, which was used in in vitro and in vivo experiments, was calculated based on $100 \%$ total solids.

\section{Cell culture and adipocyte differentiation}

The murine mesenchymal stem cell line, C3H10T1/2, was obtained from the American Type Culture Collection. Cells were cultured in Dulbecco's modified Eagle's medium (Hyclone, Marlborough, MA, USA) supplemented with $10 \%$ fetal bovine serum (Hyclone) and $1 \%$ penicillin-streptomycin solution (Hyclone) at $37^{\circ} \mathrm{C}$ in a humidified $5 \% \mathrm{CO}_{2}$ incubator.

To induce adipocyte differentiation, C3H10T1/2 cells were seeded at a density of $5 \times 10^{4}$ cells/well in 6-well plates and were grown in differentiation-inducing media containing $1 \mu \mathrm{M}$ dexamethasone (Sigma, St. Louis, MO, USA), 0.5 mM 3-isobutyl-1-methylxanthine (Sigma), $5 \mu \mathrm{g} / \mathrm{mL}$ insulin (Sigma), and $10 \mu \mathrm{M}$ troglitazone (Sigma) for the first 2 days. Then, the medium was replaced with medium containing only $5 \mu \mathrm{g} / \mathrm{mL}$ insulin (Sigma) and $10 \mu \mathrm{M}$ troglitazone (Sigma), which was replaced every 2 days for 6-7 days.

\section{Cell viability assay}

C3H10T1/2 cells were exposed to serial concentrations of WEAA during adipocyte differentiation for 7 days. For the viability test, MTT (AppliChem, Darmstadt, Germany) solution was prepared at a final concentration of $0.5 \mathrm{mg} / \mathrm{mL}$ in the media. After 4 hours, the media was removed and $100 \mu \mathrm{L}$ of dimethyl sulfoxide were added to dissolve the formazan. Next, the absorbance was measured at $540 \mathrm{~nm}$ using a SpectraMax M2/M2e Microplate Reader (Molecular Devices, San Jose, CA, USA).

\section{Oil red $O$ staining}

To measure lipid content in the cells, cells were fixed with $4 \%$ formaldehyde (Sigma) for 1 hour, stained with $0.5 \%$ Oil red O solution for 30 minutes on a shaker, and then washed with water three times. The stained cells were dissolved in $1 \mathrm{~mL} \mathrm{100 \%} \mathrm{isopro-}$ 
pyl alcohol to quantify the lipid content, and the absorbance of the solution was measured at $520 \mathrm{~nm}$ using a SpectraMax M2/M2e Microplate Reader (Molecular Devices).

Quantitative real-time polymerase chain reaction (PCR)

Total RNA from C3H10T1/2 cells was isolated with NucleoZOL (Macherey-Nagel, Düren, Germany) according to the manufacturer's protocols. cDNA was synthesized using $0.5 \mu \mathrm{g}$ total RNA with a ReverTra Ace qPCR RT Master Mix kit (Toyobo, Osaka, Japan). Synthesized cDNA was amplified with the target gene primers and THUNDERBIRD SYBR qPCR Mix (Toyobo) using the QuantStudio 3 Real-Time PCR System (Applied Biosystems, Foster City, CA, USA). Relative gene expression data were normalized to 36B4 gene expression. Primer sequences are as listed below: acidic ribosomal phosphoprotein P0 (36B4), forward 5'-AGATGCAGCAGATCCGCAT-3' and reverse 5'-GTTCTTGCCCATCAGCACC-3'; C/EBP- $\alpha$, forward 5'-GTGTGCACGTCTATGCTAAACCA-3' and reverse 5'-GCCGTTAGTGAAGAGTCTCAGTTTG-3'; adipocyte binding protein 2 (aP2), forward 5'-CACCGCAGACGACAGGAA-3' and reverse 5'-GCACCTGCACCAGGGC-3'; cluster of differentiation 36 (CD36), forward 5'-GGCCAAGCTATTGCGACAT-3' and reverse 5'-CAGATCCGAACACAGCGTA-3'; adiponectin, forward 5'-CCGGAACCCCTGGCA-3' and reverse 5'-CTGAACGCTGAGCGATACACA-3'; PPAR $\gamma$, forward 5'-TTCGCTGATGCACTGCCTA-3' and reverse 5'-GGAATGCGAGTGGTCTTCCA-3'; acetyl-CoA carboxylase (ACC), forward 5'-GCCTCAGGAGGATTTGCTGT-3' and reverse 5'-AGGATCTACCCAGGCCACAT-3'; fatty acid synthase (FAS), forward 5'-CAAGTGTCCACCAACAAGCG-3' and reverse 5'-GGAGCGCAGGATAGACTCAC-3'; stearoyl-CoA desaturase-1 (SCD-1), forward 5'-AGGTTTCCAAGCGCAGTTCC-3' and reverse 5'CTGGAAGCTCACCTCTTGGAGC-3'; UCP-1, forward 5'AAGCGTACCAAGCTGTGC-3' and reverse 5'-AGAAAAGAAGCCACAAAG-3'.

\section{Western blot}

Harvested C3H10T1/2 cells were lysed with radioimmunoprecipitation assay buffer (Thermo Scientific, Waltham, MA, USA) containing protease and phosphatase inhibitor cocktail (Thermo Scientific). Proteins ( $20 \mu \mathrm{g} /$ well $)$ were separated by $10 \%-15 \%$ so- dium dodecyl sulfate-polyacrylamide gel electrophoresis, transferred to a nitrocellulose membrane (GE Healthcare, Boston, MA, USA), and then blocked with $5 \%$ bovine serum albumin for aP2 antibody (Cell Signaling Technology, Danvers, MA, USA; \#2120) or with 5\% skim milk for the PPAR $\gamma$ (Santa Cruz Biotechnology, Santa Cruz, CA, USA; \#sc-7273), C/EBP $\alpha$ (Cell Signaling Technology, \#2295), and UCP1 (Abcam, Cambridge, MA, USA; ab10983) antibodies. Primary antibodies were incubated on the membrane overnight at $4^{\circ} \mathrm{C}$. The membrane was then washed with Tris-buffered saline-Tween 20 (TBST) three times, incubated in the secondary antibody in 5\% skim milk at room temperature for 2 hours, and then washed with TBST five times. Protein activities were detected with enhanced chemiluminescence (ECL; Amersham ECL Prime Western Blotting Detection Reagent; GE Healthcare) and protein levels were quantified with ImageJ version 1.8.0 (National Institutes of Health, Bethesda, MD, USA).

\section{Animals and experimental groups}

Zucker lean rats (Lean fa/+) and Zucker fatty (ZF) rats (fa/fa) (male, 5 weeks) were purchased from RaonBio (Yongin, Korea). The experimental groups were as follows: (1) a Zucker lean vehicle group (ZL, n=5); (2) a ZF vehicle group (ZF, n=5); and (3) a $\mathrm{ZF}+50 \mathrm{mg} / \mathrm{kg}$ WEAA group (ZF+WEAA, $\mathrm{n}=5$ ). Food (Teklad certified global 18\% protein rodent diet 2918C; Teklad, Envigo, Indianapolis, IN, USA) and water were provided ad libitum. Oral administration of vehicle or WEAA was started on the day after separation into groups and regularly performed once a day for 11 weeks. Body weight and food intake were checked once a week. Body fat mass was measured using dual energy X-ray absorptiometry (InAlyzer; Medikors, Hovedstaden, Denmark) after the animals were anesthetized with isoflurane. Fat in tissue was calculated as follows.

Fat in tissue $(\%)=$ fat mass $/$ (total body mass-bone mineral content) $\times 100$

In addition, the organs were extracted and their weights were measured. This study was performed with approval by the Animal Research Ethics Committee of KPClab (approval No. P182016).

$\mathrm{SD}$ rats (male, 5 weeks old) were purchased from Orientbio (Seongnam, Korea). After 1 week of acclimation, SD rats were randomly divided into three groups $(\mathrm{n}=9)$ in individual cages. The 
experimental groups were as follows: (1) a SD vehicle group with a normal diet (ND+water, $n=9)$; (2) SD vehicle group with a HFD+ water, $(\mathrm{n}=9)$; and (3) SD rats with a high fat diet $+200 \mathrm{mg} / \mathrm{kg}$ extract of $A$. annua group (HFD+WEAA, $n=9$ ). Water and either a normal diet (Laboratory Animal Feed; Cargill Agri Purina, Minneapolis, MN, USA) or HFD (60\% fat; Research diet, New Brunswick, NJ, USA) were provided ad libitum. Oral administration was performed once a day for 7 weeks, and body weights and food intake were measured weekly. This study was performed with a protocol approved by the Animal Research Ethics Committee of Knotus (approval No. 18-KE-253).

\section{Micro-computed tomography analysis}

Before sacrifice, micro-computed tomography (CT; vivaCT 80, Scanco Medical, Brüttisellen, Switzerland) was performed to measure total fat, which exists in the space between the origin of the second lumbar vertebrae and the fifth lumbar vertebrae. The fat volume was estimated using micro-CT software.

\section{Biochemical analysis}

After the animal was anesthetized with isoflurane, blood was collected and centrifuged at 3,000 rpm for 10 minutes. Serum was analyzed for high-density lipoprotein (HDL) cholesterol, low-density lipoprotein (LDL) cholesterol, and total cholesterol (TC), as well as triglyceride (TG), alanine aminotransferase (ALT), and aspartate aminotransferase (AST) using a blood biochemical analyzer (7180; Hitachi, Tokyo, Japan).

\section{Histological analysis}

Abdominal adipose tissues were examined for histopathological evaluation. The fixed tissues were subjected to general histological processes, such as dehydration, paraffin embedding, and cutting. Hematoxylin and eosin staining was performed, and histopathological changes were evaluated by observation using an optical microscope (Olympus BX53, Tokyo, Japan).

\section{Statistical analysis}

All experimental results are expressed as the mean \pm standard deviation, and Student t-tests were performed using GraphPad Prism software version 5.01 (GraphPad Software, San Diego, CA, USA).
One-way analysis of variance (ANOVA) and Dunnett post-test were performed to identify significant differences between animal groups, and two-way ANOVA and Bonferroni post-tests were performed for statistical analyses. A $P<0.05$ was considered statistically significant.

\section{RESULTS}

\section{Effect of WEAA on lipid accumulation in C3H10T1/2 cells}

To examine how WEAA affected lipid deposition during adipocyte differentiation in $\mathrm{C} 3 \mathrm{H} 10 \mathrm{~T} 1 / 2$ cells, we treated cells with various concentrations of WEAA for 6 days with adipocyte differentiation-inducing reagent. We confirmed that the red color of lipid droplets stained with Oil red O faded in a WEAA dose-dependent manner, suggesting that WEAA treatment remarkably inhibited lipid accumulation in C3H10T1/2 cells (Fig. 1A). To quantify the amount of intracellular lipids, stained lipid droplets were dissolved in 100\% isopropanol to measure the absorbance, and the results were compared to the untreated group. The lipid content in each group was estimated to be $91.95 \% \pm 0.32 \%$ ( $500 \mu \mathrm{g} / \mathrm{mL}$ WEAA), $79.68 \% \pm$ $0.57 \%(750 \mu \mathrm{g} / \mathrm{mL}$ WEAA $)$, and $33.26 \% \pm 0.15 \%(1,000 \mu \mathrm{g} / \mathrm{mL}$ WEAA), respectively (Fig. 1B). To confirm whether the reduction of lipid accumulation was caused by WEAA cytotoxicity, a cell viability assay was conducted using various WEAA concentrations. After growing cells to confluence, WEAA and adipocyte differentiation-inducing reagents were added to the cells for 6 days and a thiazolyl blue tetrazolium bromide (MTT) assay was performed. When compared with the untreated group, the cell survival rate increased in all WEAA-treated groups, and it was confirmed that WEAA treatment did not induce cell death (Fig. 1C). These findings suggested that WEAA attenuated adipocyte differentiation, possibly resulting in anti-obesity effects.

\section{Effect of WEAA on mRNA and protein expression of adipogenic and lipogenic genes}

As shown in Fig. 1, adipocyte differentiation and lipid accumulation were inhibited by WEAA. More specifically, we investigated whether the expression of genes related to adipocyte differentiation was affected by WEAA. The mRNA expression of genes relating to 
A

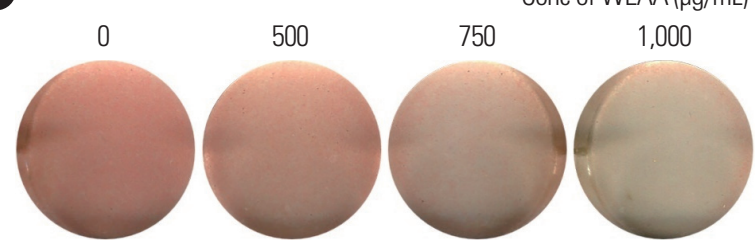

B

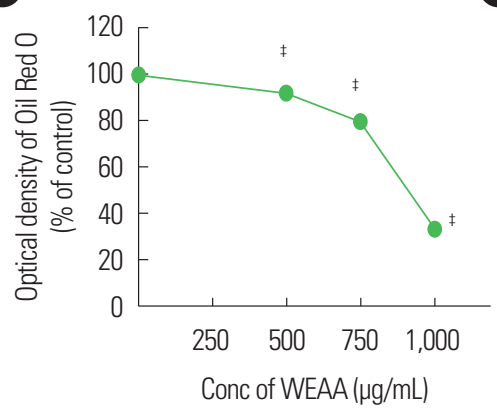

C

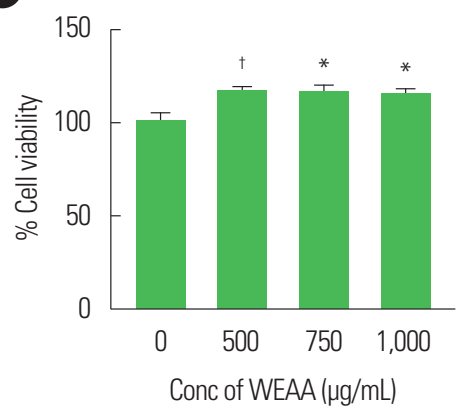

Figure 1. Water extract of Artemisia annua (WEAA) inhibits lipid accumulation in C3H10T1/2 cells. (A) Lipid levels estimated by Oil Red 0 staining in C3H10T1/2 cells exposed to different concentrations of WEAA during adipocyte differentiation. (B) Intracellular lipid contents of stained lipid droplets in C3H10T1/2 cells dissolved in 100\% isopropyl alcohol. The absorbance of the solution was measured at $520 \mathrm{~nm}$. (C) Cytotoxicity of WEAA, as evaluated using a thiazolyl blue tetrazolium bromide (MTT) assay. Results are expressed relative to the WEAA-untreated positive controls. The indicated images are representative of at least three independent experiments. Values are presented as mean \pm standard deviation and are significantly different from the WEAA-untreated positive controls, ${ }^{*} P<0.05 ;{ }^{\dagger} P<0.01$; ${ }^{\ddagger} P<0.001$. Conc, concentration.

early/late adipocyte differentiation (PPAR $\gamma, \mathrm{C} / \mathrm{EBP} \alpha, \mathrm{aP} 2$, and CD36) and of the adipokine produced primarily from adipocytes (adiponectin) were investigated with or without WEAA treatment during adipocyte differentiation. $\mathrm{C} / \mathrm{EBP} \alpha, \mathrm{aP} 2, \mathrm{CD} 36$, and adiponectin mRNA expression was increased in C3H10T1/2 cells without WEAA treatment. However, with WEAA treatment, the mRNA levels of these genes decreased in a dose-dependent manner (Fig. 2A, B). Similarly, the mRNA expression of the lipogenic-related genes ACC-1, FAS, and SCD-1 also declined with WEAA treatment (Fig. 2B). To examine the protein expression of adipogenesis-related genes during differentiation, western blotting was performed to evaluate the protein levels of $\mathrm{C} / \mathrm{EBP} \alpha, \mathrm{aP} 2$, and PPAR $\gamma$ in $\mathrm{C} 3 \mathrm{H} 10 \mathrm{~T} 1 / 2$ cells. PPAR $\gamma$ expression was slightly decreased depending on WEAA concentrations, and aP2 was predominantly reduced at the highest concentration of WEAA (a decrease of 54.32\% band intensity in contrast to WEAA-untreated cells). C/EBP $\alpha$ expression was dramatically decreased at all concentrations compared to cells without WEAA (Fig. 2C).

\section{Effect of WEAA on UCP1 mRNA and protein expression}

mRNA levels of UCP1 were increased in a concentration-dependent manner when WEAA was added to C3H10T1/2 cells during adipocyte differentiation. In particular, the two WEAA treatment groups showed a significant increase in UCP1 mRNA expression (2.15-fold with $750 \mu \mathrm{g} / \mathrm{mL} \mathrm{WEAA}$ and 2.85 -fold with $1,000 \mu \mathrm{g} / \mathrm{mL}$ WEAA) compared to the WEAA-untreated control group (Fig. 3A). We also assessed the protein expression patterns of UCP1 during
C3H10T1/2 differentiation, and found that the protein levels were slightly increased depending on the WEAA concentration (Fig. 3B). Taken together, the data in Fig. 3 suggest that increasing the expression of UCP1 might be beneficial for cells to consume intracellular energy and to defend against obesity.

\section{Effect of WEAA on the suppression of weight gain and reduction of fat mass in $\mathrm{ZF}$ rats}

Having confirmed the anti-obesity effects of WEAA in the aforementioned in vitro experiments, ZF rats were used as an obesity model to evaluate the effects of WEAA. Zucker lean and fatty rats were administered the test substance described in the Methods once a day for 11 weeks and weighed once a week. The body weights of all experimental groups increased continuously after administration. At 8 weeks, the body weight of the ZF+WEAA group (555.09 \pm $27.55 \mathrm{~g}$ ) was significantly lower than that of the $\mathrm{ZF}$ vehicle control group (593.08 $\pm 15.13 \mathrm{~g}, P=0.027)$. The significant weight loss of the $\mathrm{ZF}+\mathrm{WEAA}$ group continued until the end of the experiment. Body weights measured at 11 weeks showed weight loss rates of $8.44 \%$ in the $\mathrm{ZF}+\mathrm{WEAA}$ group compared to the $\mathrm{ZF}$ vehicle control group $(P=0.010)$ (Fig. 4A). Body fat mass was measured at a 77-day follow-up after administration using dual energy X-ray absorptiometry. The body fat mass of the ZF+WEAA group (325.38 \pm $15.95 \mathrm{~g}$ ) was significantly lower than the ZF vehicle control group $(372.34 \pm 34.31 \mathrm{~g})(P=0.026)$. In addition, lean mass was decreased in the WEAA group compared with the ZF vehicle control group, but the change was not statistically significant (Fig. 4B). We also 
A

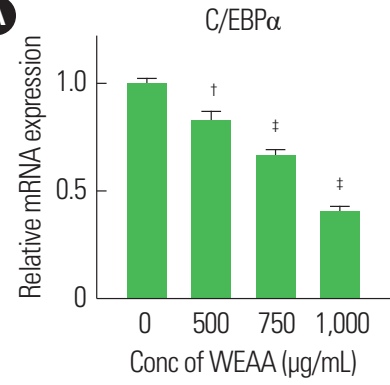

B

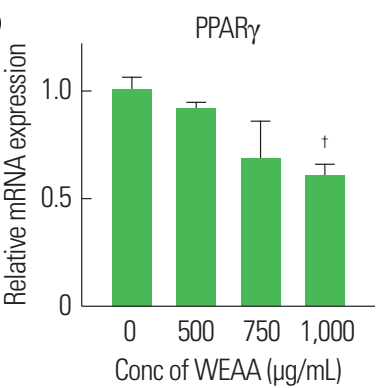

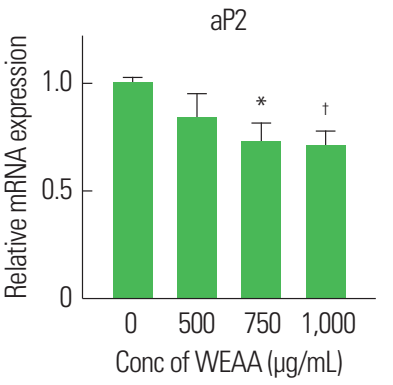

ACC-1

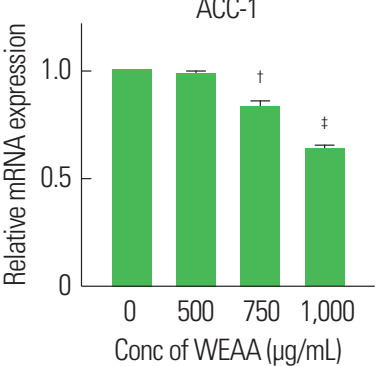

CD36

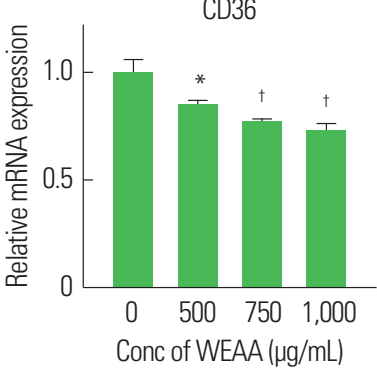

FAS

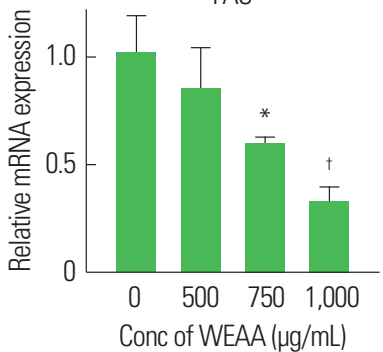

Adiponectin

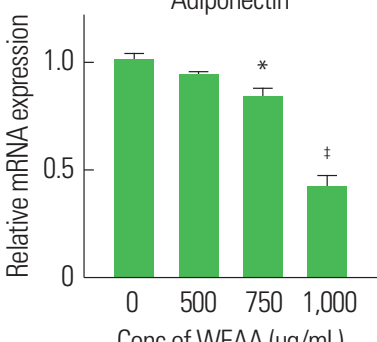

SCD-1

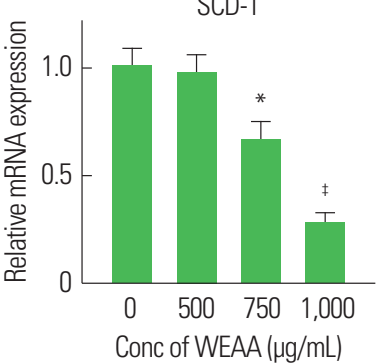

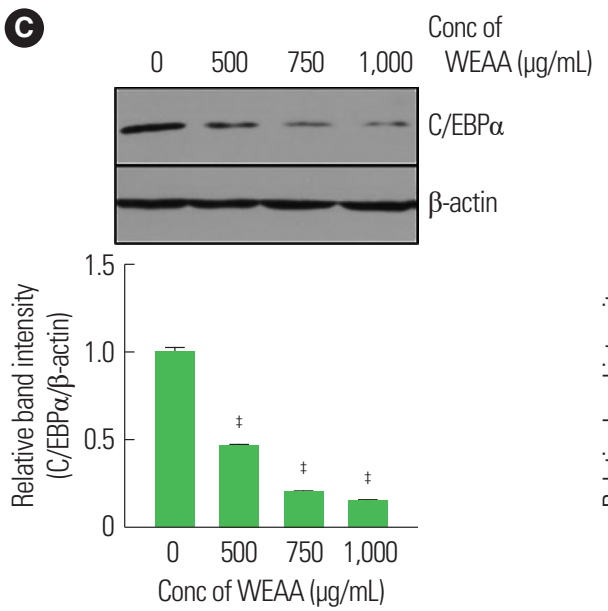
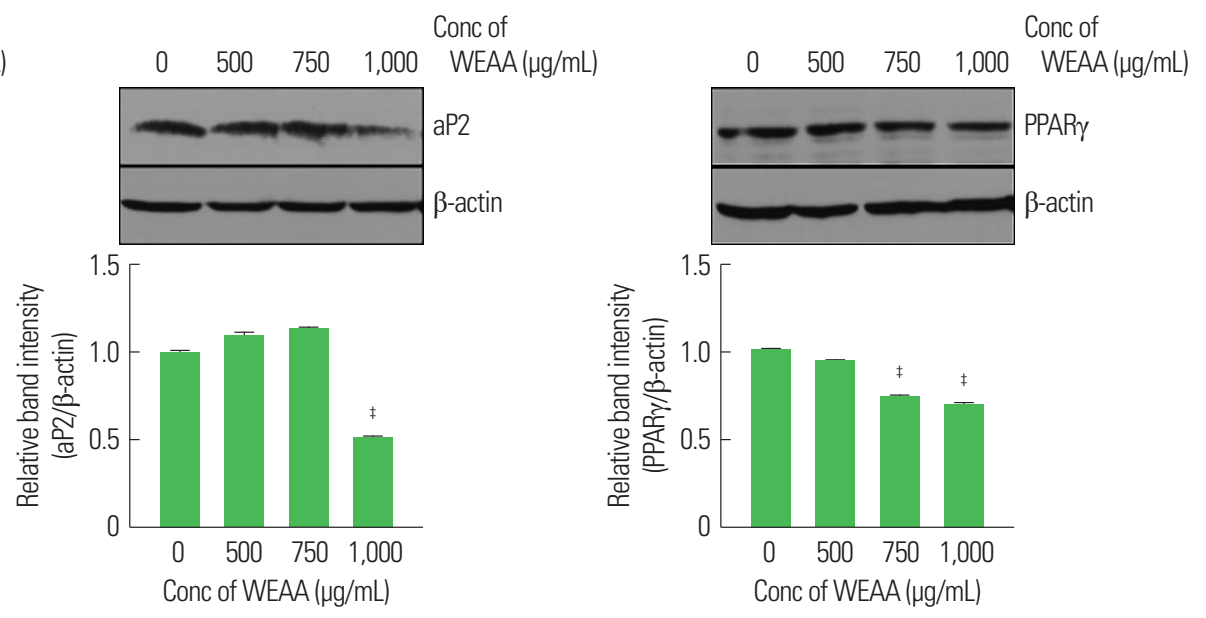

Figure 2. Water extract of Artemisia annua (WEAA) suppresses mRNA and protein expression of adipogenic and lipogenic factors. C3H10T1/2 cells were treated with the indicated concentration of WEAA upon adipocyte differentiation. (A, B) mRNA expression of adipogenic and lipogenic genes, as evaluated by real time polymerase chain reaction. Relative mRNA expression was normalized to 36B4 gene expression. (C) CCAAT/enhancer-binding proteins (C/EBP $\alpha$ ), adipocyte binding protein (aP2), and peroxisome proliferation-activated receptor (PPARy) protein expression, as detected by Western blotting. Proteins were isolated using radioimmunoprecipitation assay buffer containing protease and phosphatase inhibitor cocktail. $\beta$-actin was used as a loading control. Band intensity was calculated using ImageJ software (data not shown). The results are representative of at least three independent experiments. Values are presented as mean \pm standard deviation and are significantly different from the WEAA-untreated positive controls, ${ }^{*} P<0.05$; ${ }^{\dagger} P<0.01$; ${ }^{\ddagger} P<0.001$. Conc, concentration; $\mathrm{CD} 36$, cluster of differentiation 36 ; ACC, acetyl;-CoA carboxylase; FAS, fatty acid synthase; SCD-1, stearoyl-CoA desaturase-1.

analyzed the relative weight of fat tissue to confirm the effects of WEAA on anatomical aspects. Relative interscapular brown fat was increased in the ZF+WEAA (1.26-fold) groups compared to that in the $\mathrm{ZF}$ vehicle group (Fig. 4C). Moreover, it was confirmed that the amount of fat in the tissue of the ZF+WEAA group was lower than that in the ZF vehicle control group (Fig. 4D).
Effect of WEAA on body weight gain, fat tissue weight, and adipocyte size in HFD-induced obesity SD rats

We also investigated the effects of WEAA on a HFD-induced obesity model. SD rats were fed a 60\% HFD for 49 days to induce obesity. The body weights of all HFD-induced obesity groups were significantly higher than that of the normal diet group from the start date of the test to the end of the test $(P<0.001)$. The HFD+ 

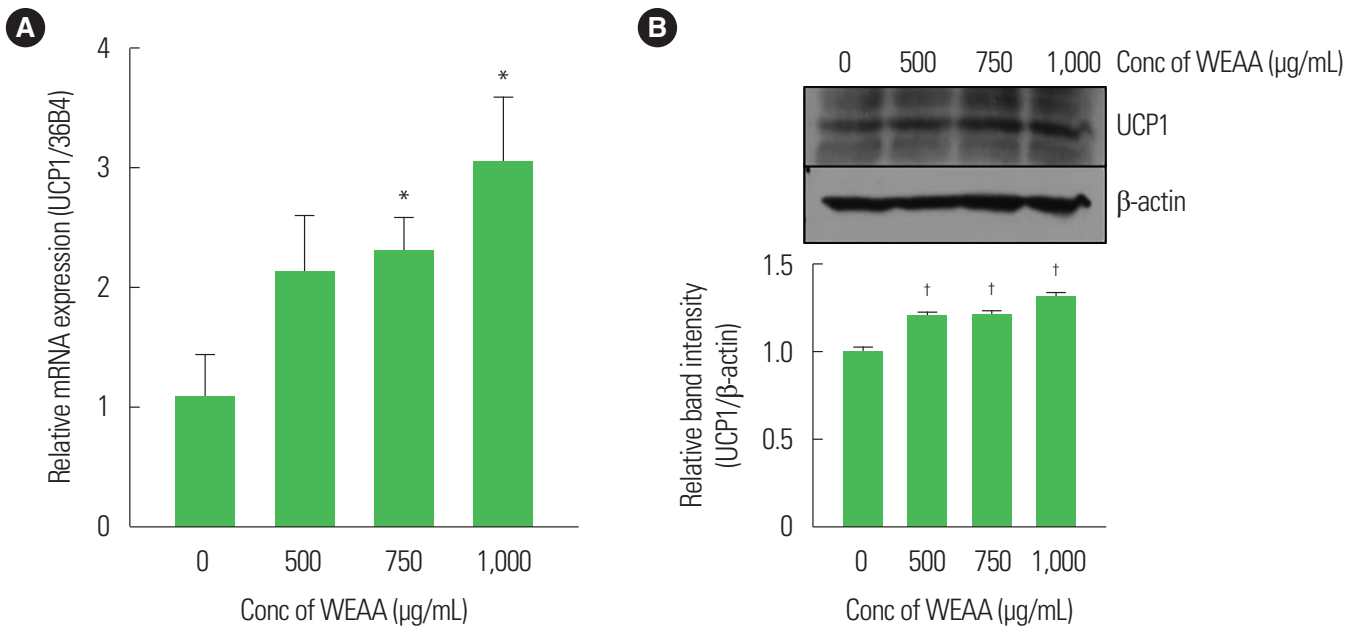

Figure 3. Water extract of Artemisia annua (WEAA) increases uncoupling protein 1 (UCP1) mRNA and protein expression in C3H10T1/2 cells. (A) UCP1 gene expression, as evaluated by real-time polymerase chain reaction. Relative mRNA expression was normalized to 36B4 gene expression. (B) UCP1 protein expression, as detected by Western blotting. $\beta$-actin was used as a loading control. Band intensity was calculated using Image J software. The results are representative of at least three independent experiments. Values are presented as mean \pm standard deviation and are significantly different from the WEAA-untreated positive controls, ${ }^{*} P<0.05 ;{ }^{\dagger} P<0.001$. Conc, concentration.
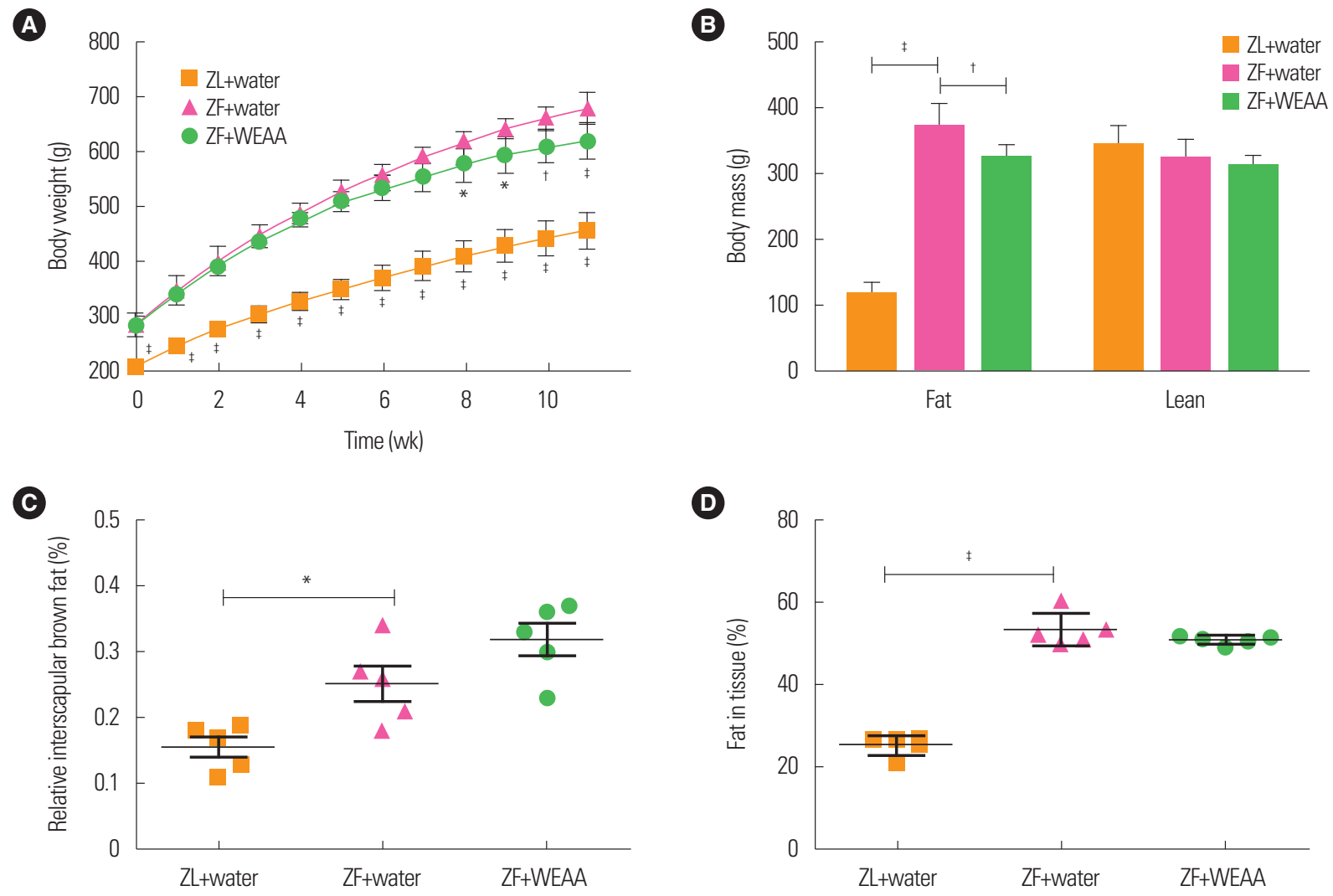

Figure 4. Water extract of Artemisia annua (WEAA) reduces body weight gain in Zucker fatty (ZF) rats. Zucker lean (ZL) and ZF rats were orally administered WEAA (50 $\mathrm{mg} / \mathrm{kg}$ ) or water once a day for 11 weeks. (A) Body weight, measured weekly. (B) Body fat mass, as measured using dual energy X-ray absorptiometry after inhalation anesthesia with isoflurane. (C) Relative interscapular brown fat, calculated as the percent of organ weight/body weight. (D) Fat in tissue, calculated by the fat mass divided by the subtraction of the bone mineral content from the total body mass. The experimental groups are as follows: ZL+water, ZF+water, and ZF+WEAA (50 mg/kg). Values are presented as mean \pm standard deviation $(n=5)$. All statistical analyses are calculated compared to the ZF+water group. $(A, B)$ Two-way analysis of variance $(A N O V A)$ followed by the Bonferroni post-test was used; (C, D) one-way ANOVA followed by the Dunnett post-test was used. ${ }^{*} P<0.05 ;{ }^{\dagger} P<0.01 ;{ }^{\ddagger} P<0.001$. 
WEAA group tended to lose weight compared to the HFD vehicle group during the entire experimental period, although there was no significant difference (Fig. 5A). In the micro-CT images, in- creased abdominal fat was observed in the HFD group compared to the normal diet group, whereas abdominal fat was decreased in the HFD+WEAA group compared to the HFD vehicle group (Fig.
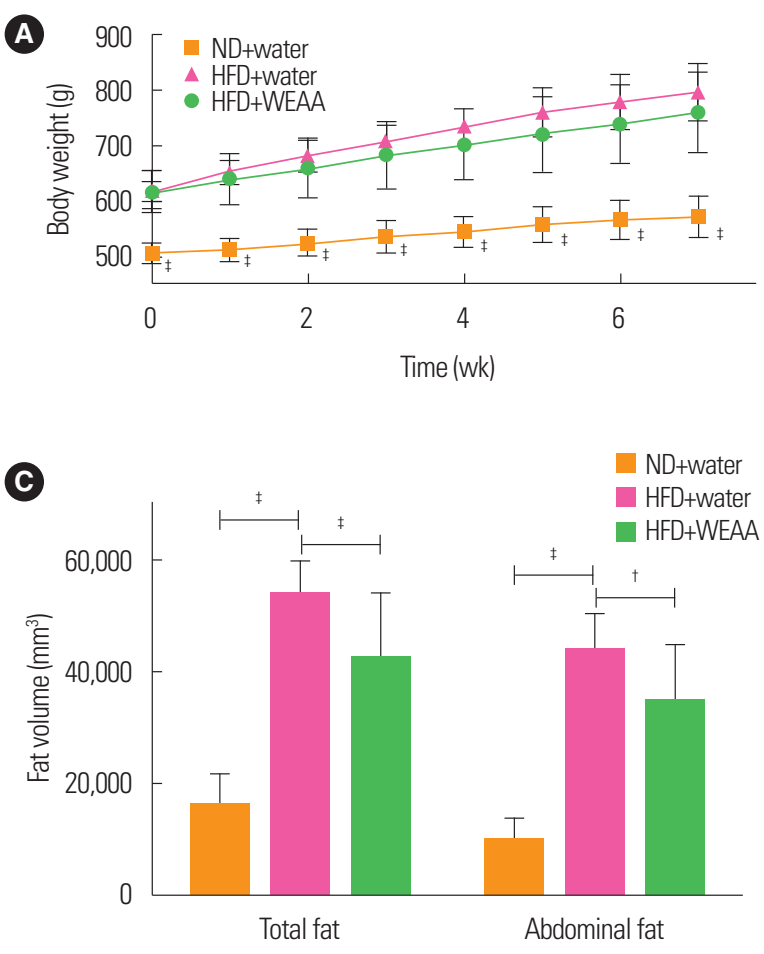

Figure 5. Water extract of Artemisia annua (WEAA) suppresses body weight gain and decreases fat tissue weight and adipocyte size in Sprague Dawley (SD) rats with high-fat diet (HFD)-induced obesity. SD rats were orally administered WEAA $(200 \mathrm{mg} / \mathrm{kg}$ ) or water once a day for 7 weeks. SD rats were fed a normal diet or a $60 \%$ HFD during the experimental period. (A) Body weight, measured weekly. The experimental groups are as follows: (1) SD vehicle group with a normal diet (ND+water, $n=9)$, (2) SD vehicle group with a HFD (HFD+water, $n=9)$, and (3) SD rats with a HFD+200 mg/kg WEAA group (HFD+WEAA, $n=9$ ). $(\mathrm{B}, \mathrm{C})$ The micro-computed tomography (CT) image is representative of each experimental group and fat volume was calculated using the microCT software. (D) Relative organ weight, calculated as percent of organ weight/body weight. (E) Adipocyte diameter, calculated by measuring at least five adipocyte globules in each abdominal tissue of the experimental group. H\&E images are representative of each group $(\times 100)$. Scale bars indicate $50 \mu \mathrm{m}$. Values are presented as mean \pm standard deviation $(n=9)$. All statistical analyses are calculated compared to the HFD+water group. (A, C) Two-way analysis of variance (ANOVA) followed by the Bonferroni post-test was used; (D, E) One-way ANOVA followed by Dunnett post-test was used. ${ }^{*} P<0.05 ;{ }^{\dagger} P<0.01 ;{ }^{\ddagger} P<0.001$.
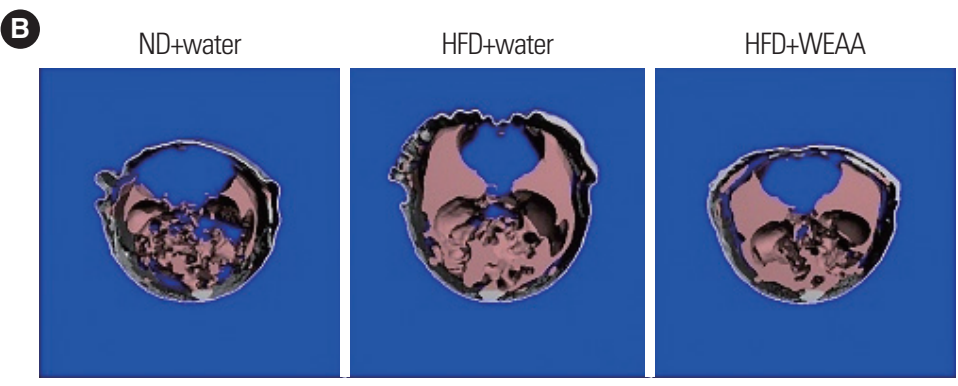

(D)
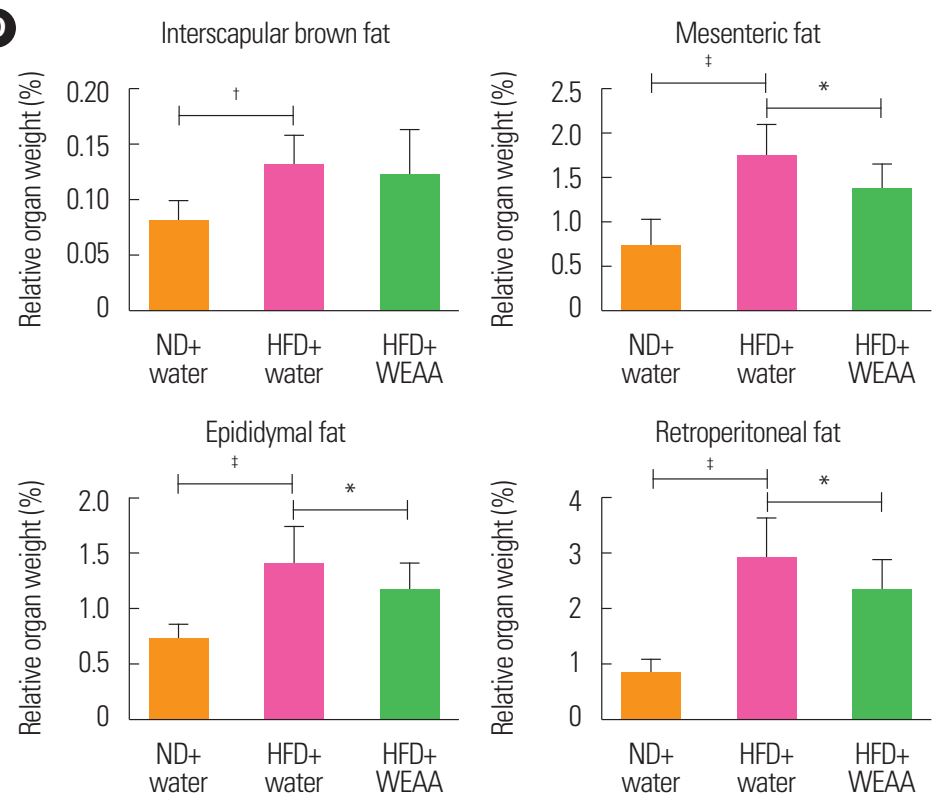

$\boldsymbol{E}$
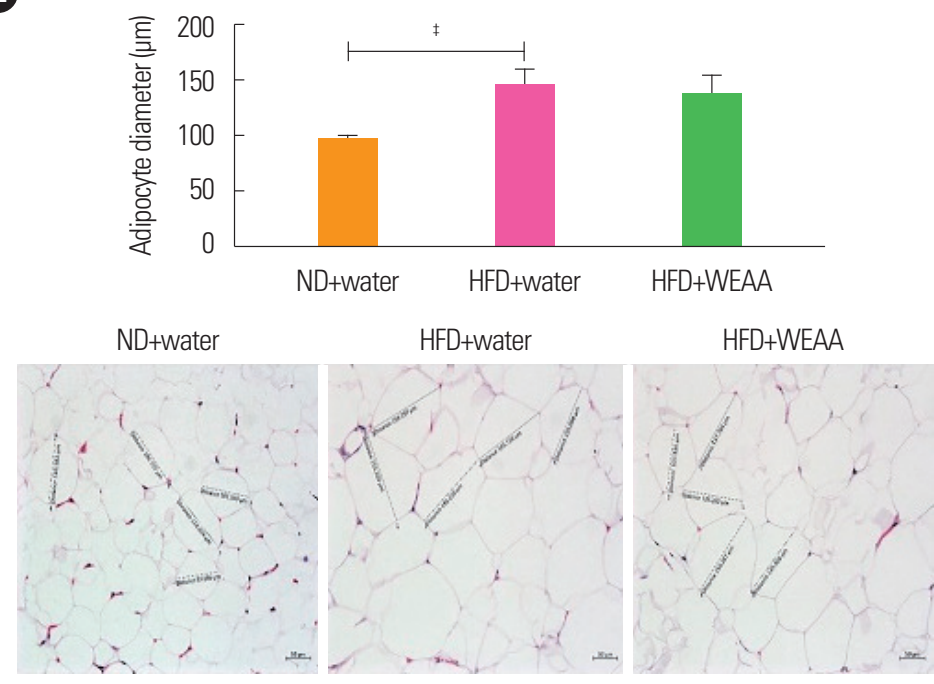
5B). The total fat volume of all HFD groups was significantly higher than that of the normal diet group, and the total fat volume of the WEAA group was significantly lower than that of the HFD group $(P<0.001)$. In the same manner, abdominal fat volume was increased in both of the HFD groups compared to the normal diet group, but was reduced in the HFD+WEAA group compared to the HFD vehicle group $(P<0.01)$ (Fig. $5 C)$. We also examined the effects of WEAA on adipose tissue and found that the weights of mesenteric, epididymal, and retroperitoneal fat significantly decreased in the HFD+WEAA group compared to the HFD vehicle group $(P<0.05)$ (Fig. 5D). In contrast to the white fat tissue, the weight of interscapular brown fat in the WEAA administration group was similar to the brown fat of the HFD vehicle group. Histological analysis could determine the change in adipocyte cell size depending on the substrate treatment. Compared to the normal diet group, the abdominal adipocyte size of the HFD vehicle group increased by $49.95 \mu \mathrm{m}$. In the WEAA-treated group, adipocyte diameter decreased by $10.02 \mu \mathrm{m}$ (Fig. 5E) compared to the HFD vehicle group. Images in Fig. 5E show reduced adipocyte size with WEAA administration compared to the HFD vehicle group.

\section{Effect of WEAA on blood serum biochemical factors in}

\section{HFD-induced obesity SD rats}

Biochemical analysis of blood serum revealed that HDL cholesterol levels in the HFD+WEAA group were slightly greater (16.73 \pm $3.33 \mathrm{mg} / \mathrm{dL})$ than in the HFD vehicle group $(16.19 \pm 2.79 \mathrm{mg} / \mathrm{dL})$, but there was no significant difference (Fig. 6A). LDL cholesterol (Fig. 6B), TC (Fig. 6C), and TG (Fig. 6D) levels were lower in the HFD+WEAA group than in the HFD vehicle group. In particular, LDL cholesterol, and TG levels in the HFD+WEAA group were significantly reduced by $44.3 \%$ and $44.8 \%$, respectively $(P<0.01$, $P<0.001)$. Even though there was no statistical difference, the TC level was decreased by $19.24 \%$ in the HFD+WEAA group. Additionally, ALT (Fig. 6E) and aspartate AST (Fig. 6F) levels were increased in the HFD group compared to the normal diet group. WEAA treatment of the HFD group caused considerable reduction of ALT $(33.9 \pm 12.4 \mathrm{U} / \mathrm{L})$ and AST $(110.6 \pm 17.5 \mathrm{U} / \mathrm{L})$ when compared to the HFD vehicle group (ALT, 42.6 $\pm 5.8 \mathrm{U} / \mathrm{L}$; AST, $167.3 \pm 25.2 \mathrm{U} / \mathrm{L})$. Administration of WEAA in HFD-induced obese rats restored ALT and AST serum levels to those of normal rats without HFD administration.
A

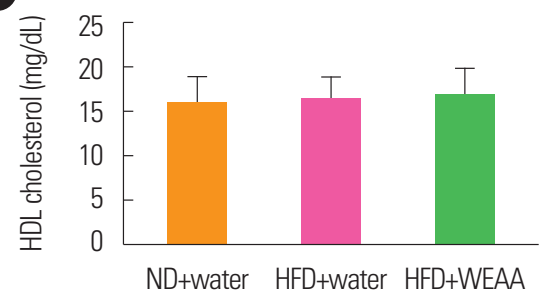

(D)

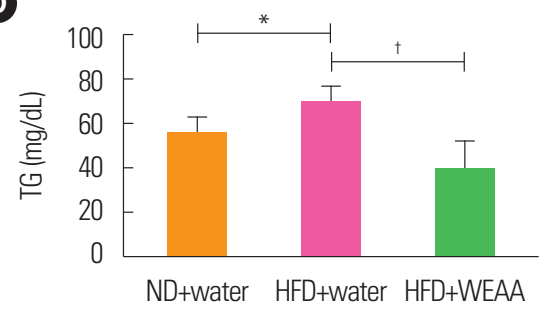

B

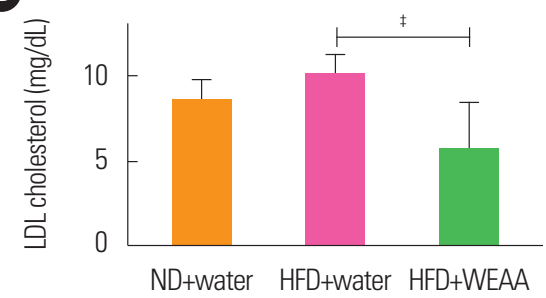

E

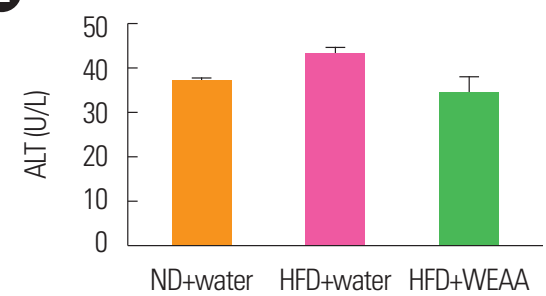

C

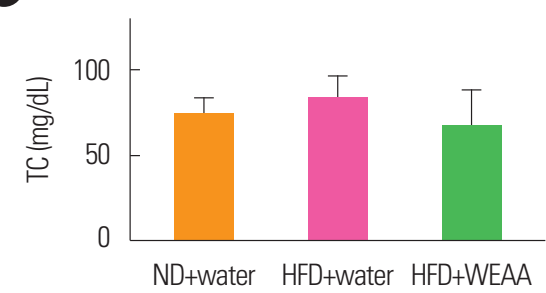

F

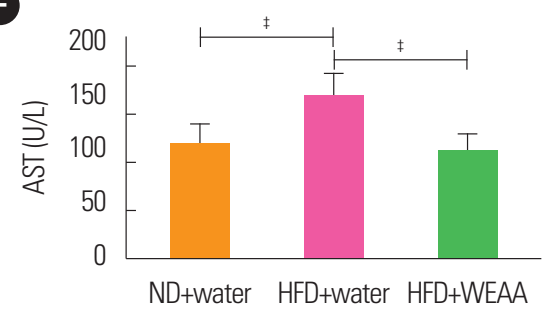

Figure 6. Water extract of Artemisia annua (WEAA) affects the biochemical index of high-fat diet (HFD)-induced obesity in Sprague Dawley (SD) rats. SD rats were orally administered WEAA (200 mg/kg) or water once a day for 7 weeks. SD rats were fed a normal diet or a 60\% HFD during the experimental period. The experimental groups are as follows: (1) SD vehicle group with a normal diet (ND+water, $n=9$ ); (2) SD vehicle group with a HFD (HFD+water, $n=9$ ); and (3) SD rats with a HFD+200 mg/kg WEAA group (HFD+WEAA, $n=9$ ). After the animal was anesthetized with isoflurane, blood was collected and centrifuged at 3,000 rpm for 10 minutes. Serum was analyzed for (A) high-density lipoprotein (HDL) cholesterol, (B) low-density lipoprotein (LDL) cholesterol, and (C) total cholesterol (TC), as well as (D) triglyceride (TG), (E) alanine aminotransferase (ALT), and (F) aspartate aminotransferase (AST). Values are presented as mean \pm standard deviation. One-way analysis of variance followed by Dunnett post-test was performed for statistical analysis. ${ }^{*} P<0.05 ;{ }^{\dagger} P<0.01 ;{ }^{\ddagger} P<0.001$. 


\section{DISCUSSION}

A. annua, mainly grown in East Asia, has long been used as a medicinal herb with antipyretic and antibacterial properties. ${ }^{17,18}$ There are also a number of reports using $A$. annua to control obesity. ${ }^{19,20}$ When HFD-induced obese mice and rats are fed with alcoholic extracts of young leaves of $A$. annua, the rate of weight gain is slowed and the blood levels of TG and TC decrease. ${ }^{21,22}$ The WEAA used in this study has previously shown anti-obesity activity in HFD-induced obese mice. ${ }^{23}$ In the early stages of adipocyte differentiation, the expression of $\mathrm{C} / \mathrm{EBP} \beta$ and $\mathrm{C} / \mathrm{EBP} \delta$ induce the expression of PPAR $\gamma$, which subsequently promotes $\mathrm{C} / \mathrm{EBP} \alpha$ expression. ${ }^{24}$

Sterol regulatory element-binding protein 1c (ADD1/SREBP1c) is mainly expressed at high levels in white adipose tissue, brown adipose tissue, and liver, and is expressed very early in the differentiation process of adipocytes. ${ }^{25}$ Target genes of ADD1/SREBP1c include FAS, lipoprotein lipase, glycerol-3-phosphate acyltransferase 1, SCD1, SCD2, and ACC, which are enzymes responsible for the regulation of fatty acid metabolism. ${ }^{26}$ The discovery that the addition of a PPAR $\gamma$ ligand can recover blockage of adipocyte differentiation mediated by dominant-negative ADD1/SREBP1c indicates that the role of $\mathrm{ADD} 1 / \mathrm{SREBP} 1 \mathrm{c}$ is to promote endogenous ligand or activator production of PPAR $\gamma .{ }^{26}$ Inhibition of adipocyte differentiation has been reported for artemisinic acid and artesunate, a single small molecule derived from A. annua. ${ }^{19,27}$ Artemisinic acid reduces gene expression of PPAR $\gamma$ and subsequent expression of $\mathrm{C} / \mathrm{EBP} \alpha$ and its targets aP2, lipoprotein lipase, $\mathrm{CD} 36$, and liver $\mathrm{X}$ receptor. While artemisinic acid does not change C/EBP $\beta$ levels, it reduces $\mathrm{C} / \mathrm{EBP} \delta$ gene $\mathrm{mRNA}$ levels and TG accumulation in adipocytes. ${ }^{19}$ Artesunate also reduces the expression of C/EBP- $\alpha$, PPAR-g, and FAS during adipocyte differentiation, and inhibits fat accumulation and TG synthesis. ${ }^{27}$

Our study demonstrated that WEAA decreased the expression levels of PPAR $\gamma$, which plays a central role in adipocyte differentiation, and subsequently reduced the expression of $\mathrm{C} / \mathrm{EBP} \alpha, \mathrm{aP} 2$, and CD36, ultimately regulating differentiation stages of adipogenesis. In addition, WEAA reduced the expression of the FAS, SCD1, SCD2, and ACC enzymes that regulate fatty acid metabolism under the influence of PPAR $\gamma$. Together, these results suggest that WEAA is involved in regulation of fatty acid metabolism as well as fat cell dif- ferentiation, thus suppressing TG synthesis and overall obesity.

The Zucker rat is an experimental animal model in which obesity is induced by leptin deficiency at the receptor level, a peptide hormone derived from adipocytes that has strong anti-obesity and energy consumption-promoting effects. ${ }^{23}$ In this study, we demonstrated that WEAA suppressed fat differentiation and fat accumulation in the ZF rat model, as well as the HFD-induced obesity rat model. In contrast to the tendency for total body fat to decrease, brown fat did not differ between the test and control groups in either animal model. In particular, the total fat and abdominal obesity of the test group that ingested WEAA was significantly decreased compared to the vehicle control group in the HFD-induced obesity rat model. Typical white fats such as mesenteric, epididymal, and retroperitoneal fat decreased significantly in the test group, while brown fat between the scapula remained unchanged between the groups.

Brown fat has great utility for fatty acid oxidation and TCA activity and leads to metabolism to promote maximal cellular respiration and calorific emission. ${ }^{28} \mathrm{UCP} 1$ promotes energy production by inducing heat production in brown or beige fat cells and reducing mitochondrial respiratory efficiency. ${ }^{28}$ WEAA suppressed the expression of the mast cell differentiation-promoting gene, but increased the expression of UCP1, which is involved in the conversion of white to brown fat during adipogenic differentiation of C3H10T1/2 cells, a mouse mesenchymal stem cell line (Fig. 3). Also, rats in the HFD-induced obesity group that ingested WEAA for 8 weeks showed upregulation of the UCP1 gene in white adipose tissue (Supplementary Fig. 1). Therefore, WEAA may activate total fat browning and slow weight gain by promoting brown fat thermogenesis in HFD-induced obese animals. UCP1 promotes oxidation of fatty acids in the mitochondria through a process of reducing oxidized Nicotinamide adenine dinucleotide+/flavin adenine dinucleotide and activating the beta-oxidation cycle and the Krebs cycle to activate fatty acid outflow. ${ }^{29}$ UCP1 deficiency enhances hyperlipidemia in apolipoprotein $\mathrm{E}$ mice at a temperature of $30^{\circ} \mathrm{C}$, which causes fatty liver disease. ${ }^{30}$ In addition, UCP1 deficiency increases the translocation of fatty acids into the liver and results in TG accumulation in the liver.

High levels of LDL cholesterol were found in $37.5 \%$ of nonalcoholic fatty liver patients, and low levels of HDL cholesterol were found in $19.5 \%$. This suggests that hyperlipidemia, which includes 
excessive TG and cholesterol in the blood, is closely related to the occurrence of nonalcoholic fatty liver diseases. ${ }^{31}$ The blood levels of HDL cholesterol did not change, but LDL cholesterol was significantly decreased in the test group that consumed WEAA compared to the control group that did not ingest $A$. annua in the HFDinduced obesity model. In particular, TG levels in the blood of the test group were reduced, and the liver function indicators AST and ALT were restored to normal values. These results indicated that WEAA could improve liver function by preventing obesity and the occurrence of fatty liver due to a HFD.

\section{CONFLICTS OF INTEREST}

The authors declare no conflict of interest.

\section{ACKNOWLEDGMENTS}

This work was supported by the Korea Institute of Planning and Evaluation for Technology in Food, Agriculture, Forestry and Fisheries (IPET) through High Value-added Food Technology Development Program and by Korean Food Research Institute through Food Function Evaluation Support Program, funded by Ministry of Agriculture, Food and Rural Affairs (MAFRA; No. 2017100491).

This work was also supported by grant no. 04-2018-0250 from the Seoul National University Hospital Research Fund.

\section{AUTHOR CONTRIBUTIONS}

Study concept and design: DK, BH, and SHK; acquisition of data: EYC and CYP; analysis and interpretation of data: EYC, CYP, SHH, and SHK; drafting of the manuscript: DK and SHK; critical revision of the manuscript: SJP, DK, BH, and SHK; statistical analysis: EYC, CYP, and SHH; obtained funding \& administrative, technical, or material support: SJP, DK, and SHK; and study supervision: $\mathrm{BH}$ and $\mathrm{SHK}$.

\section{SUPPLEMENTARY MATERIALS}

Supplementary Figure 1 can be found via https://doi.org/10. $7570 /$ jomes20097.

\section{REFERENCES}

1. Bornstein SR, Ehrhart-Bornstein M, Wong ML, Licinio J. Is the worldwide epidemic of obesity a communicable feature of globalization? Exp Clin Endocrinol Diabetes 2008;116 Suppl 1:S30-2.

2. Kahn SE, Hull RL, Utzschneider KM. Mechanisms linking obesity to insulin resistance and type 2 diabetes. Nature 2006; 444:840-6.

3. LeRoith D, Novosyadlyy R, Gallagher EJ, Lann D, Vijayakumar A, Yakar S. Obesity and type 2 diabetes are associated with an increased risk of developing cancer and a worse prognosis; epidemiological and mechanistic evidence. Exp Clin Endocrinol Diabetes 2008;116 Suppl 1:S4-6.

4. Sainaghi PP, Castello L, Bergamasco L, Carnevale Schianca GP, Bartoli E. Metabolic characteristics of glucose intolerance: the critical role of obesity. Exp Clin Endocrinol Diabetes 2008; 116:86-93.

5. Van Gaal LF, Mertens IL, De Block CE. Mechanisms linking obesity with cardiovascular disease. Nature 2006;444:875-80.

6. Jo J, Gavrilova O, Pack S, Jou W, Mullen S, Sumner AE, et al. Hypertrophy and/or hyperplasia: dynamics of adipose tissue growth. PLoS Comput Biol 2009;5:e1000324.

7. Rosen ED, Spiegelman BM. Molecular regulation of adipogenesis. Annu Rev Cell Dev Biol 2000;16:145-71.

8. Rangwala SM, Lazar MA. Transcriptional control of adipogenesis. Annu Rev Nutr 2000;20:535-59.

9. Rosen ED, Walkey CJ, Puigserver P, Spiegelman BM. Transcriptional regulation of adipogenesis. Genes Dev 2000;14:1293307.

10. Darlington GJ, Ross SE, MacDougald OA. The role of C/EBP genes in adipocyte differentiation. J Biol Chem 1998;273: 30057-60.

11. Evans RM, Barish GD, Wang YX. PPARs and the complex journey to obesity. Nat Med 2004;10:355-61.

12. Del Mar Gonzalez-Barroso M, Ricquier D, Cassard-Doulcier AM. The human uncoupling protein-1 gene (UCP1): present status and perspectives in obesity research. Obes Rev 2000;1: 61-72.

13. Garlid KD, Jabůrek M, Jezek P. The mechanism of proton 
transport mediated by mitochondrial uncoupling proteins. FEBS Lett 1998;438:10-4.

14. Kopecký J, Hodný Z, Rossmeisl M, Syrový I, Kozak LP. Reduction of dietary obesity in aP2-Ucp transgenic mice: physiology and adipose tissue distribution. Am J Physiol 1996; 270(5 Pt 1):E768-75.

15. Kopecký J, Rossmeisl M, Hodný Z, Syrový I, Horáková M, Kolárová P. Reduction of dietary obesity in aP2-Ucp transgenic mice: mechanism and adipose tissue morphology. Am J Physiol 1996;270(5 Pt 1):E776-86.

16. Hard to swallow. Nature 2007;448:105-6.

17. Efferth T, Dunstan H, Sauerbrey A, Miyachi H, Chitambar $\mathrm{CR}$. The anti-malarial artesunate is also active against cancer. Int J Oncol 2001;18:767-73.

18. Li Y. Qinghaosu (artemisinin): chemistry and pharmacology. Acta Pharmacol Sin 2012;33:1141-6.

19. Lee J, Kim MH, Lee JH, Jung E, Yoo ES, Park D. Artemisinic acid is a regulator of adipocyte differentiation and C/EBP $\delta$ expression. J Cell Biochem 2012;113:2488-99.

20. Hwang DI, Won KJ, Kim DY, Yoon SW, Park JH, Kim B, et al. Anti-adipocyte differentiation activity and chemical composition of essential oil from Artemisia annua. Nat Prod Commun 2016;11:539-42.

21. Kim KE, Ko KH, Heo RW, Yi CO, Shin HJ, Kim JY, et al. Artemisia annua Leaf extract attenuates hepatic steatosis and inflammation in high-fat diet-fed mice. J Med Food 2016;19: 290-9.

22. Song Y, Lee SJ, Jang SH, Kim TH, Kim HD, Kim SW, et al. Annual wormwood leaf inhibits the adipogenesis of 3t3-11 and obesity in high-fat diet-induced obese rats. Nutrients 2017;9: 554.
23. Baek HK, Shim H, Lim H, Shim M, Kim CK, Park SK, et al. Anti-adipogenic effect of Artemisia annua in diet-inducedobesity mice model. J Vet Sci 2015;16:389-96.

24. Cao Z, Umek RM, McKnight SL. Regulated expression of three C/EBP isoforms during adipose conversion of 3T3-L1 cells. Genes Dev 1991;5:1538-52.

25. Kim JB, Spiegelman BM. ADD1/SREBP1 promotes adipocyte differentiation and gene expression linked to fatty acid metabolism. Genes Dev 1996;10:1096-107.

26. Kim JB, Wright HM, Wright M, Spiegelman BM. ADD1/ SREBP1 activates PPARgamma through the production of endogenous ligand. Proc Natl Acad Sci U S A 1998;95:4333-7.

27. Jang BC. Artesunate inhibits adipogeneis in 3T3-L1 preadipocytes by reducing the expression and/or phosphorylation levels of C/EBP- $\alpha$, PPAR- $\gamma$, FAS, perilipin A, and STAT-3. Biochem Biophys Res Commun 2016;474:220-5.

28. Kozak LP, Anunciado-Koza R. UCP1: its involvement and utility in obesity. Int J Obes (Lond) 2008;32 Suppl 7(Suppl 7):S32-8.

29. Cortez-Pinto H, Machado MV. Uncoupling proteins and nonalcoholic fatty liver disease. J Hepatol 2009;50:857-60.

30. Kataoka N, Takeuchi T, Kusudo T, Li Y, Endo Y, Yamashita H. Lack of UCP1 stimulates fatty liver but mediates UCP1-independent action of beige fat to improve hyperlipidemia in Apoe knockout mice. Biochim Biophys Acta Mol Basis Dis 2020; 1866:165762.

31. Nakahara T, Hyogo H, Yoneda M, Sumida Y, Eguchi Y, Fujii H, et al. Type 2 diabetes mellitus is associated with the fibrosis severity in patients with nonalcoholic fatty liver disease in a large retrospective cohort of Japanese patients. J Gastroenterol 2014;49:1477-84. 